\title{
DETECTION OF ANTIBIOTIC RESISTANCE IN Pseudomonas aeruginosa ISOLATES WITH SPECIAL REFERENCE TO METALLO B-LACTAMASES FROM A TERTIARY CARE HOSPITAL IN WESTERN INDIA
}

\author{
ANGADI K.M. ${ }^{*}{ }^{*}$, KADAM M. ${ }^{2}$, MODAK M.S. ${ }^{2}$, BHATAVDEKAR S.M. ${ }^{2}$, DALAL B.A. ${ }^{2}$, JADHAVVAR S.R. ${ }^{2}$, \\ TOLPADI A.G. ${ }^{2}$ THAKKAR V. ${ }^{2}$ AND SHAH S.R. ${ }^{2}$
}

${ }^{1}$ Pad. Dr. D.Y. Patil Medical College and Research Centre, Pimpri, Pune-411018, MS, India.

2Bharati Vidyapeeth Medical College and Hospital, Pune-411043, MS, India.

*Corresponding Author: Email- kalpanaangadi@yahoo.com

Received: August 07, 2012; Accepted: August 14, 2012

\begin{abstract}
-
Background: Pseudomonas aeruginosa is an important cause of morbidity and mortality in hospitalised patients. The prevalence of multidrug resistant Pseudomonas aeruginosa strains including resistance to Carbapenems has been an increasing cause of concern. Hence this study was undertaken to know the resistance pattern of Pseudomonas aeruginosa to various anti-pseudomonal antibiotics and also to detect Metallo $\beta$ lactamase production in them.

Methodology: Pseudomonas aeruginosa isolates from various clinical samples were tested. Antibiotic sensitivity testing was carried out by Kirby- Bauer method according to CLSI guidelines and detection of Metallo- $\beta$-lactamase production was carried out by Imipenem EDTA combined disc method.

Results: 125 clinical isolates of Pseudomonas aeruginosa were tested. The resistance pattern to various antibiotics were- Amikacin (18.4\%), Gentamycin (63.2\%), Netilmycin (32.8\%), Ceftazidime (74.4\%), Ciprofloxacin (60\%), Imipenem (21.6\%), Piperacillin (54.4\%), Piperacillin+tazobactum (45.6\%), Polymyxin-B (20\%), Colistin (15.2\%), Tobramycin $(55.2 \%)$, Levofloxacin $(41.6 \%)$, Carbenicillin (51.2\%). $21.6 \%$ of the isolates were resistant to Imipenem and 6 out of 27 i.e $22.2 \%$ were positive for Metallo $\beta$ lactamase production. Conclusions: Amikacin, Carbapenems, Colistin and Polymyxin are the main drugs to treat multidrug resistant Pseudomonas aeruginosa and Metallo $\beta$ lactamase detection has to be done to identify resistance to Carbapenems.
\end{abstract}

Key words- Pseudomonas aeruginosa, Metallo $\beta$ lactamases

Citation: Angadi K.M., et al. (2012) Detection of Antibiotic Resistance in Pseudomonas aeruginosa Isolates with Special Reference to Metallo B-Lactamases from A Tertiary Care Hospital in Western India. International Journal of Microbiology Research, ISSN: 0975-5276 \& E-ISSN: 0975-9174, Volume 4, Issue 7, pp.-295-298.

Copyright: Copyright@2012 Angadi K.M., et al. This is an open-access article distributed under the terms of the Creative Commons Attribution License, which permits unrestricted use, distribution and reproduction in any medium, provided the original author and source are credited.

\section{Introduction}

Pseudomonas aeruginosa (Ps. aeruginosa) is the most common opportunistic pathogen of all Pseudomonas species. The importance of this species derives from the wide spread distribution of its strains in nature, their resistance to many antibacterial compounds and the number of virulence factors produced by them [1]. Pseudomonas are very rich source of plasmids carrying genes for a wide variety of functions, such as resistance to antibiotics and miscellaneous antibacterial agents including chemical agents such as metals and inorganic anions, resistance to bacteriophages, bacteriocins. In addition to its resistance to antimicrobial compounds, it is able to produce various virulence properties in the form of enzymes and toxins. The arsenal of the organism has several extracellular proteases among which the most prominent are alkaline proteases, esterase and cytotoxin [2].
In normal, healthy hosts, infection is usually associated with events that disrupt or by pass protection provided by the epidermis e.g. burns, puncture wounds, use of contaminated needles by IV drug abusers, eye trauma with contaminated contact lenses. The result is infection of the skin, bone, heart or eye. Ps. aeruginosa is a notable cause of nosocomial infections of the respiratory and urinary tracts, wounds, blood stream and even the central nervous system [3]. In an immunocompromised patient, such infections are severe and frequently life threatening $[1,2]$.

Ps. aeruginosa shows considerable degree of natural resistance to antibiotics. With the need to treat antibiotic resistant pseudomonas, newer drugs like carbapenem were introduced. These carbapenem are the last resources for treatment of multidrug resistant gram negative infections because of their broad antimicrobial activity and stability against most common beta lactamases $[3,4]$. Metallo $\beta$ - 
lactamases $(\mathrm{MBL})$ are the enzymes which can hydrolyze all beta lactam (except Aztreoman) including Carbapenems. They belong to the Class $b$ of Ambler Classification of $\beta$ lactamases wherein $\mathrm{Zn}$ $\left(\mathrm{Zn}^{+2}\right)$ is used to break the amide bond [4]. Various reports are present wherein variable resistance to the carbapenem have been mentioned [5-14]. Because of the widespread resistance of Pseudomonas to various antibiotics including the Carbapenem, this study was undertaken to know the antimicrobial susceptibility pattern with special reference to MBL production by Ps. aeruginosa strains in our hospital.

\section{Material and Methods}

The study was approved by ethical committee of same institute.

\section{Study Site}

Present study was carried out in Bharati Vidyapeeth's Medical college and Hospital Pune-Maharashtra India.125 consecutive isolates of $P$ s. aeruginosa were collected over a period of 6 months from June 2010 to December 2010, from various clinical specimens received in the department of microbiology. All samples were processed and identified by standard conventional methods $[2,15]$. Antibiotic sensitivity testing was done by Kirby- Bauer Disc diffusion method and interpreted according to CLSI (Clinical Laboratory standard institute) guidelines [16]. Antibiotic sensitivity testing was done for the following antibiotics -Gentamycin(10ug), Tobramycin (10ug), Netilmycin(30ug), Amikacin(30ug), Ciprofloxacin(5ug), Levofloxacin(5ug), Cephotaxime, Ceftazidime(30ug), Piperacillin (100ug), Piperacillin /tazobactum(100/10ug), Imipenem(10ug), Colistin(10 ug), Polymyxin B(300ug). Those isolates which exhibited resistance or reduced susceptibility for Imipenem disc were selected for further detection and phenotypic confirmation of MBL production by Imipenem-EDTA (Ethylene diamine tetra acetic acid) combined disc test method.

\section{Detection of Metallo $\beta$-lactamases by Imipenem-EDTA Com- bined Disc Test}

Detection of MBL production by pseudomonal isolates was done by Imipenem EDTA Combined Disc method as described by Lee, et al. [17]. The selection criteria for MBL detection was reduced susceptibility to Imipenem (inhibition zone diameter less than $16 \mathrm{~mm}$ ). A $0.5 \mathrm{M}$ EDTA solution was prepared by dissolving $186.1 \mathrm{gm}$ of disodium EDTA $2 \mathrm{H} 2 \mathrm{O}$ in $1000 \mathrm{ml}$ of distilled water and $\mathrm{pH}$ was adjusted to 8.0 using $\mathrm{NaOH}$. The mixture was sterilized by autoclaving. Test organism was inoculated on Muller Hinton agar. Two 10ug Imipenem disc was placed on the surface of the agar plate and appropriate amount of EDTA solution was added to one of the disc to obtain the desired concentration of 750ug. The inhibition zone of Imipenem and Imipenem EDTA were compared after 16-18 hours of incubation at $35^{\circ} \mathrm{C}$. If there was an increase in the inhibition zone of more than $7 \mathrm{~mm}$ than the Imipenem disc alone, it was considered as MBL positive. ATCC 27853 Ps. aeruginosa was used as negative control.

\section{Results and Observations}

125 non-repetitive $P$ s. aeruginosa isolates from various clinical specimens were tested. Out of the 125 specimens, 45 were from Surgical wards, 19 from Medical wards, 18 from the Intensive care units, 21 from combined Orthopaedics, OBGY, ENT and Ophthalmology wards, 17 from OPD and 5 from Paediatric ward (Fig. 1)

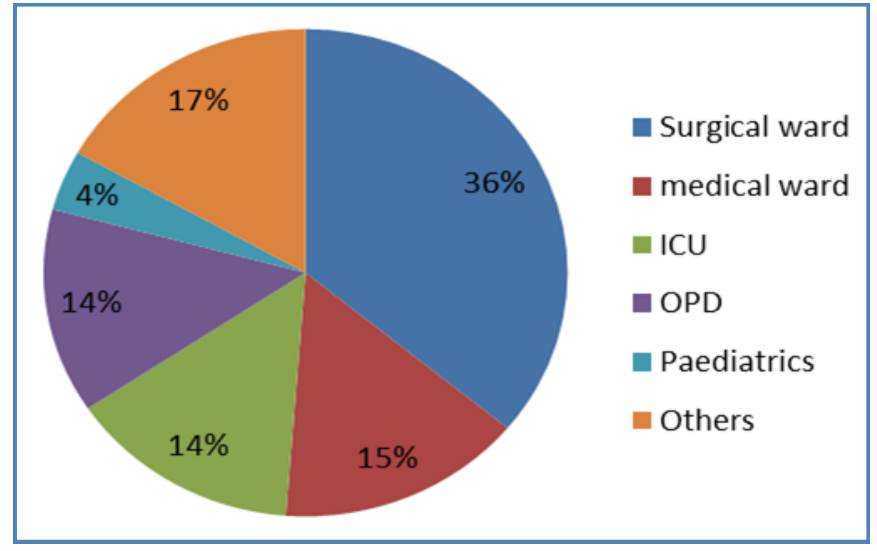

Fig. 1- Distribution of $P$ s. aeruginosa from various wards Others- ENT (13), Ortho, Ophthalmology (3), Burns (4). OBG

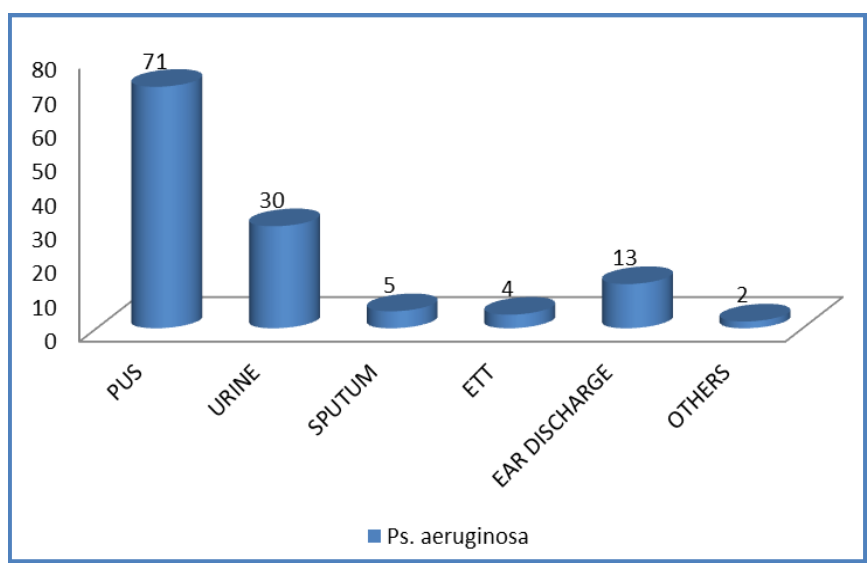

Fig. 2- Distribution of $P$ s. aeruginosa from various clinical samples

The most common source of Ps. aeruginosa were Pus samples from wounds and from urine (Fig. 2)

The antibiotic sensitivity testing results indicated the following rates of resistance, Amikacin (18.4\%), Imipenem (21.6\%), Polymyxin (20\%), Colistin (15.2\%), Netilmycin (32.8\%), Piperacillin (54.4\%), Tobramycin $(55.2 \%)$, Carbenicillin $(51.2 \%)$, Piperacillin+tazobactum (45.6\%), Levofloxacin $(41.6 \%)$, Ciprofloxacin $(60 \%)$, Gentamycin $(63.2 \%)$, Ceftazidime $(74.4 \%)$, Cefotaxime $(75.2 \%)$ which is shown in Fig. 3.

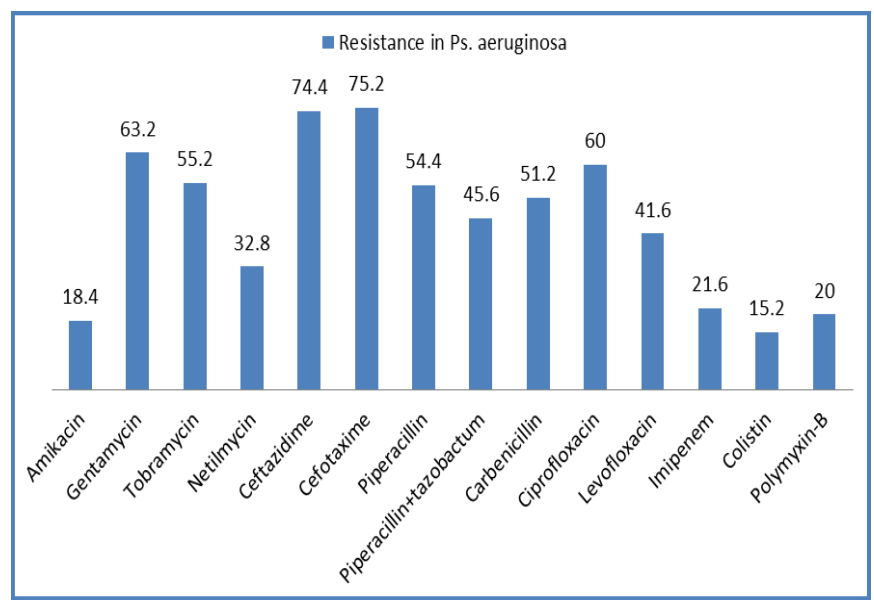

Fig. 3- Resistance pattern of $P$ s. aeruginosa to various antibiotics 
Resistance to Imipenem was seen in $21.6 \%$ of the isolates. Detection of MBL production was done by Imipenem EDTA Combined Disc method and 6 out of 27 isolates $(22.2 \%)$ were positive for production of MBL

\section{Discussion}

Ps. aeruginosa is currently one of the most frequently isolated nosocomial pathogen and the infections due to this organism are often difficult to treat due to resistance to various antibiotics. The general resistance is due to a combination of factors. It is intrinsically resistant to antimicrobial agents due to low permeability of its cell wall. It has the genetic capacity to express a wide repertoire of resistance mechanism. It has become resistant through mutation in chromosomal genes which regulate resistance genes and it can acquire additional resistance genes from other organisms via plasmids, transposons and bacteriophages [18].

In the present study, the resistant pattern of clinical isolates of $P S$. aeruginosa studied showed lower resistance to Amikacin (18.4\%), Imipenem (21.6\%), Colistin (15.2\%), Polymyxin (20\%) and increasing resistance to Netilmycin (32.8\%) Piperacillin+tazobactum $(45.6 \%)$ and high resistance to Quinolones $(40-60 \%)$ and third generation Cephalosporins (>70\%). Among the aminoglycosides, resistance to Amikacin was seen in $18.4 \%$ of the isolates in our study, while lower rate of resistance were reported from Pakisthan, $6.73 \%$ by Nadeem, et al. [19] and $8 \%$ by Nadeem and Qasmi, et al. [20], a resistance of $21 \%$ was reported by Farida, et al. (2010) [21], Jamshaid, et al. (2008) reported 24\% resistance [22], 25\% in Nagaveni, et al. also reported similar finding in 2010 [10]. Relative findings also reported from various studies which showed higher rates of resistance i.e. $42.8 \%$ by Murugan, et al. [13], $50 \%$ by Viren, et al. study [23] and 73\% in Tehran by Horeih Saderi, et al. [9], $74 \%$ in Behera, et al. study [24,25]. In 2012 higher rate of resistance has been reported by Madhu Sharma, et al. i.e. $91.2 \%$ [26], $96.6 \%$ by Awari, et al. [12] and $97 \%$ by Bhale Rao, et al. [27].

Our study showed $63.2 \%$ resistance to Gentamycin, while the study by Nadeem, et al. showed lower resistance i.e.12.9\% [19] and $21.6 \%$ by Paul, et al. in their studies [28].

In 2010 Murugan, et al. study showed moderate resistance i.e. $42.8 \%$ to Gentamycin [13], comparatively we found that India, Pakisthan and Iran studies showed higher rate of resistance to Gentamycin- aminoglycosides [9,10,12,21,23,26,27,29], while in aminoglycoside-Netilmycin showed fair susceptibility rates i.e. $67.2 \%$ in our study and similar finding has been reported from $\mathrm{Pa}$ kisthan by Nadeem, et al. i.e. 90\% [19] but higher rate of resistance were reported by Viren, et al. from India (60\%) [23]. Resistance to Tobramycin was seen in $55.2 \%$ of isolates in our study, while lower rates of resistance was observed with Nicholas, et al. 1\% [30], Farida, et al. 30\% [21], Paul, et al. I 25.5\% [28], 44\% in Nagaveni, et al. I study [10], higher rates of $66 \%$ was seen in study by Viren, et al. [23] and Pittout, et al. [14], 71.4\% in Murugan, et al. study [13] and $98 \%$ in Awari, et al. study [12].

Resistance to the third generation Cephalosporins (Cefotaxime and Ceftazidime) was seen $75 \%$ in our study. Lower rates were observed by Nicholas, et al. (6\%), Nadeem, et al. (10.9\%), Nadeem and Qasmi, et al. (16\%), Paul, et al. (19.6\%), Farida, et al. (38\%) $[19,20,21,28,30]$. Higher resistance to third generation cephalo- sporins has been reported by Pittout, et al. (59\%), Viren, et al. $(67.86 \%)$, Prajapathi, et al. (68\%) and $(70 \%)$ by Behera, et al. [14, $23,25,29]$. In 2010 from India very high rate of resistance to third generation cephalosporins have been recorded $(>90 \%)$ by Bhale Rao, et al. and (96-100\%) by Awari, et al. [12,27]. Higher rate of susceptibility to third generation cephalosporins were reported by Nadeem, et al. (88.7\%), Farida, et al. (73\%), by Nadeem and Qasmi, et al. (89\%), Nicholas, et al. (85\%), Paul, et al. (76.5\%) [19$21,28,30]$. We detected comparatively lower resistance to Levofloxacin $(41.6 \%)$ in India than other reported studies i.e. (62.5\%) by Viren, et al., (71\%) Prajapathi, et al. and Behera, et al. both in their studies showed $(57.1 \%)$ by Murugan, et al. [13,23,25,29].

Resistance to Piperacillin was seen in (54.4\%) isolates in our study and lower rate of resistance were reported in other studies by Nicholas, et al. (4\%), Nadeem, et al. (10.8\%) and (15.7\%) in Paul, et al., $(20 \%)$ by Pittout, et al. (35\%) by Farida, et al. study [14,19,21, $28,30]$, whereas higher rates of resistance was seen with Behera, et al. study $75 \%$ [24] 73\% in Horeih Saderi, et al. study [9], 85\% in Awari, et al. study [12], 88.8\% in Madhu Sharma, et al. study [26], $100 \%$ in Murugan, et al. study [13]. Resistance to Piperacillin+ tazobactum combination was seen in $45.6 \%$ of our isolates, lower rates of $9.4 \%$ was observed in Nadeem, et al. [19] and a slightly higher resistance i.e 54\% was seen in Prajapathi, et al. study [29] and $59 \%$ in Behera, et al. study [25]. Resistance to the Imipenem were seen in $(21.6 \%)$ of isolates in our study, while lower rates were seen with Farida, et al. (3\%), Nicholas, et al. (9\%), Paul, et al. (9.8\%), Nadeem, et al. (9.9\%) $[19,21,28,30]$ while higher rate of resistance were reported by Nagaveni, et al. (32\%), (71.4\%) by Murugan, et al., Prajapathi, et al. (59\%), (69\%) Horeih Saderi, et al., $(55 \%)$ by Awari, et al. in their studies $[9,10,12,13,29]$.

Among all isolated imipenem resistance strains of $P$ s. aeruginosa $(n=27)$ from present study, we found ( $n=7$ i.e. $22.2 \%)$ strains were $\mathrm{MBL}$ producers, detected by phenotypic detection method of $\mathrm{MBL}$ production. We found reasonably similar rate of MBL producer's strains of Ps. aeruginosa from other studies i.e. (20.8\%) by Nagaveni, et al. [24] and (28\%) by Anuradha, et al. [25] from India and $(24.2 \%)$ by Nam Hee Ryoo, et al. from Korea [26]. Varsha Gupta, et al. reported MBL production (84\%), Bhale Rao, et al. (67\%) and $72 \%$ by Fereshteh shaheheraghi, et al. [31,27,32]. $16 \%$ of the Imipenem resistant isolates were positive for MBL production in Rajput Anuradha, et al. study [33]. MBL production was seen in $50 \%$ of $P$ s. aeruginosa isolates in Dey, et al. study [5]. MBL production was seen in $20.8 \%$ of the isolates in Nagaveni, et al. study [10]. $70 \%$ were positive for MBL in Murugan, et al. study [13], 46\% were positive for MBL production in Pittout, et al. study [14], In Manoharan, et al. study $42.6 \%$ were found to be MBL positive [35] and in Deeba Bashir, et al. study $13.42 \%$ of Ps. aeruginosa were resistant to Imipenem and $11.66 \%$ were positive for MBL production [36].

Prevalence of MBL producing clinical isolates of Pseudomonas species have been continuously reported globally with some disparity in the rates of resistance. As MBL producing Pseudomonas species poses therapeutic problems in hospitals, it is better to understand the mechanism and spread of such multidrug resistant strains. This is our initial step towards controlling the spread of MDR (Multidrugresistant) strains by detecting their incidence in our hospital. 
Limitations of this study is that this is a retrospective study so we could not implement some statistical analysis for finding some qualitative data, we analyzed this data from clinical samples received in microbiology laboratory from various wards and OPD so is subjected to sampling bias and phenotypic confirmation of MBL should be further characterized by molecular detection methods by which we can find predominant MDR strain of Ps. aeruginosa. To defeat our limitations, we are implementing genotypic characterization of such MDR isolates and also collecting appropriate demographic data and clinical information concerning such isolates from our isolates by which we can locate out the source of infection as well as determine the mechanism of resistance of MDR strains.

\section{Conclusion}

Increase in antibacterial resistance in Ps. aeruginosa is a cause of concern. So, continuous monitoring of bacterial resistance trends should be done and therapy should be based on antibacterial susceptibility results. Infection control programme and policies should be vigorously pursued in our health care facilities as well as antibiotic prescription regulation to cope with the upsurge of Ps. aeruginosa resistance to various antibiotics. Thus proper antibiotics policy and measures to restrict the indiscriminate use of carbapenems should be taken to minimize the emergence of this MBL producing pathogen, whose spread would leave no option to treat gram negative infections.

\section{References}

[1] Greenwood D., Slack C.B. Richard, Peutherer F. John (2002) Medical Microbiology. 16th Edition., 282.

[2] Forbes B.A., Sahm D.F., Weissfeld A.S. (2007) Bailey and Scotts Daignostic Microbiology., 20th Edition, 343.

[3] Thomson J.M. and Bonomo R.A. (2005) Current Opinion Microbiol., 8(5), 18-24.

[4] Mandell Gerald L., Bennett John E., Dolin Raphael (2010) Principles and Practice of Infectious Diseases., 7th Ed., 281-284.

[5] Dey A., Bairy I. (2007) American Thoracic Medicine, (2), 52-57.

[6] Hemlata V., Uma Sekar and Vijaylaxmi Kamat (2005) Indian J. of Med. Research, (122), 148-152.

[7] Ami Varaiya, Nikhil Kulkarni, Manasi Kulkarni, et al. (2008) Indian J. of Medical Research, (127), 398-402.

[8] Dong fang, XU Xi-Wei, Song Wen-Qi, et al. (2008) Chinese Medical Journal, 121(17), 1611-1616.

[9] Horieh Saderi, Hosein Lotfalipour, Parviz Owlia, Hassan Salimi (2010) Lab. Medicine., 41(10), 609.

[10]Nagaveni S., Rajeshwari H., Ajaykumar Oli, Patil S.A. and Kelmani Chandrakanth R. (2010) The Bioscan., (20), 251-253.

[11]De A.S., Kumar S.H., Bhaveja S.M. (2010) Indian Journal. Critical Care Med., (14), 217-219.

[12]Awari Abhijit and Nighute Sunita (2012) International Journal of Biomedical Research, 3(1), 32-35.

[13]Murugan S., Bakkiya Lakshmi R., Umadevi P. and Mani K.R. (2010) Int. J. of Microbiological Research, 1(30), 123-128.
[14]Johann D. Pitout, Daniel B. Gregson, Laurent Poirel (2005) Journal of Clinical Microbiology, 43(7),3129.

[15]Collee J.G., Diguid J.P., Fraser A.G. (1996) Mackie \& Mc Cartney Practical Medical Microbiology., 14th Edition, 131-149.

[16]Clinical Laboratory Standards Institute (2007) Performance Standard for Antimicrobial Susceptibility Testing: Seventeenth Informational Supplement, 27(1), M100-S17.

[17]Lee K., Lim Y.S., Yong D., Yum J.H., Chong Y. (2003) J. Clin. Microbiology., 41(10), 4623-4629.

[18]Lambert P.A. (2001) J. Royal Soc. Med., (41), 22-26.

[19]Nadeem Sajjad Raja and Nishi Nihar Singh (2007) J. Microbial. Immunol. Infect. (40), 45-49.

[20]Nadeem S.G., Qasmi S.A., Afaque F., et al. (2009) Pakisthan BJMP, 2(4), 35-39.

[21]Farida Anjum and Asif Munir (2010) African Journal of Microbiology Research, 49(100), 1005-1012.

[22]Jamshaid A.K., Zafar I., Saeed U.R., et al. (2008) Pakistan Journal of Pharm. Sci., 21(3), 311-315.

[23]Viren A.J., Somsuvra B.G., Kamlesh R.P., et al. (2008) Indian Journal of Pharmacology, 40(5), 230-234.

[24]Benjamin Behera, Anupam Das, Purva Mathur, et al. (2008) Indian Journal of Medical Research, 128(3), 324-325.

[25]Behera B., Mathur P., Das A., Kapil A., et al. (2008) Indian Journal of Medical Microbiology, 26(3), 233-237.

[26]Madhu Sharma, Sarita Yadav, Uma Chaudary (2010) Lab. Physicians, (2), 14-16.

[27]Bhalerao D., Roushani S., Kinikar A.G. (2010) Pravara Med. Rev., 5(3),16-19.

[28]Paul D. Brown and Amicetus Izundu (2004) Pan. Am. J. Public Health, 16 (2):125-30.

[29]Prajapati S.B., Vegad M.M., Mehta S.J., et al. (2011) Journal of Cell and Tissue Research, II(1), 2601-2604.

[30]Nicholas Ttroillet, Mathew H. Samore and Yehuda Carmeli (1997) Clinical Infectious Diseases, 25, 1094-8.

[31]Varsha Gupta, Nidhi Singla and Jagdish Chander (2008) Indian J. Med. Res., (128), 671-672.

[32]Fereshteh S., Vajiheh S.N., Mohammed Mahdi Feizabadi (2010) New Microbiologica. (33), 243-248.

[33]Rajput, Anuradha, Saxena, Rishi Singh Kaleshwar Prasad (2010) Burn Care Research, 31(2), 264-268.

[34]Nam Hee Ryoo, Jung Sook Ha, Dong Seok Jeon, et.al. (2010) Korean J. Clin. Microbio. 13(4), 169-172.

[35]Manoharan A., Chatterjee S., Mathai (2010) Indian J. Medical Microbiology, (28), 241-244.

[36]Deeba B., Manzoor A. T., Bashir A. F., et al. (2011) African Journal of Microbiology Research, 5(2), 164-172. 